\title{
Conversatorios, talleres y editatonas: tecnopolítica feminista y redes de aprendizaje en México
}

\author{
Discussions, workshops and editatonas: feminist technopolitics \\ and networked learning in Mexico
}

\section{Claudia Pedraza}

Universidad La Salle, Ciudad de México, México

claudia.pedrazaAlasalle

\section{César Rodriguez}

Universidad Autónoma Metropolitana, Ciudad de México, México

carcanodcorreo.cua.uam.mx

\section{Resumen}

El propósito de este artículo es analizar las redes de aprendizaje surgidas de los procesos de apropiación tecnológica que promueven los colectivos de tecnopolítica feminista en México. El marco analítico retoma los conceptos de apropiación digital y aprendizaje en red desde una perspectiva tecnopolítica, destacando la reticularidad de los procesos y las prácticas sociales. A partir de la revisión de los carteles de difusión de actividades de los colectivos durante más de dos años, el enfoque de análisis intersecta las relaciones entre las temáticas y modalidades registradas, desde las tradiciones del Análisis de Redes Sociales y la construcción de redes semánticas. Los hallazgos revelan un ecosistema con talleres, conversatorios y editatonas como eventos clave en una red que configura procesos de reflexión, apropiación e incidencia tecnológica, a través de usos que reconocen la agencia de las mujeres; socializaciones dialógicas y orientadas a fomentar la participación activa en las TIC.

Palabras clave: Apropiación tecnológica, aprendizaje en red, tecnopolítica, ciberfeminismo, TIC, Análisis de Redes Sociales.

\begin{abstract}
The purpose of this article is to analyze the complexity of learning networks that emerge during the process of technological appropriation promoted by feminist collectives in Mexico. The analytical approach considers the concepts of digital appropriation and networked learning from a technopolitical perspective, as a networked processes and social practices. For this reason, the body of research, namely the posters of events disseminated and occurred over more than two years, was registered as a relational database of topics and modalities finally analyzed in a hybrid construction composed by a Social Network Analysis (SNA) and a semantic networks outlook. Successfully, the major findings took shape as a rich ecosystem between reflection, appropriation and influence with discussions, workshops and editatonas as key modalities. Additionally, a network of centralities was revealed as a configuration of uses that acknowledges women's agency, socializations that arise from dialogic activities, and significances oriented towards active participation in social practices enabled by ICT.
\end{abstract}

Keywords: Technological appropriation, networked learning, technopolitics, cyberfeminism, ICT, Social Network Analysis. 


\section{Introducción}

Durante décadas, la relación de las mujeres y las tecnologías ha sido problematizada desde posturas que coinciden en señalar la exclusión femenina de los saberes, las prácticas y los significados de lo tecnológico (Haraway, 1995; Plant, 1998; Hawthorne \& Klein, 1999; Braidotti, 2002;Castaño, 2005; Zafra, 2005; Wajcman, 2006 Hargittai, 2007). No obstante, el surgimiento de las Tecnologías de la Información y la Comunicación (TIC) posibilitó usos y re-significaciones para la población femenina, con un potencial subversivo que ha transformado su usual exclusión. La creación de blogs, medios digitales, performances, instalaciones, producciones audiovisuales, activismos mediados por las plataformas sociodigitales y otras acciones a través de las TIC han permitido que mujeres y colectivos visibilicen problemáticas, reivindiquen derechos y se organicen para desafiar las relaciones de poder que atraviesan sus vidas.

Pero este potencial subversivo no está al alcance de todas debido a las diferencias estructurales que condicionan el acceso, uso y apropiación de las tecnologías. La evidencia más notoria de dichas condicionantes es la brecha digital de género', entendida como las diferencias de participación de mujeres y hombres en las TIC (Pagola, 2010). Según la Unión Internacional de Telecomunicaciones (UIT, 2017), esta brecha se manifiesta en todo el mundo: en los países catalogados como desarrollados el uso de Internet es de $79.9 \%$ en mujeres y $82.2 \%$ en varones; el porcentaje disminuye drásticamente en los países con el menor índice de desarrollo, donde acceden a las TIC el $14.1 \%$ de mujeres y $21 \%$ de varones. Los factores que determinan la brecha digital de género están ligados a otras diferencias estructurales como las desigualdades económicas, el acceso a la educación tanto básica como especializada en tecnologías, y las prácticas emergentes de violencia digital, las cuales configuran el acercamiento de la población femenina a estas tecnologías (ONU, 2015; UNESCO, 2019).

Ante las diferencias estructurales que limitan la participación femenina en los entornos digitales y en los procesos que a través de ellos se generan, han surgido una cantidad notable de esfuerzos formales e informales para fomentar el acercamiento de las mujeres a las tecnologías. De manera particular, en este texto interesa analizar la propuesta de las redes de tecnopolítica feminista en la Ciudad de México, que en los últimos años han cobrado notoriedad como parte del movimiento ciberfeminista en la región, en el marco de los nuevos horizontes de reflexión convocados en torno a las miradas posibles de las tecnologías digitales (Ortiz Henderson y Garay Cruz, 2015). La hipótesis es que estas organizaciones configuran redes de aprendizaje que promueven la apropiación tecnológica de las mujeres a partir de modalidades y temáticas cuya intención es transformar su exclusión para convertirlas en participantes activas de los entornos digitales.

\section{Marco teórico}

\subsection{La apropiación tecnológica desde la perspec- tiva de género}

A nivel internacional, fomentar el acceso, uso y apropiación tecnológica de las mujeres se establece como una tarea necesaria que, en gran parte, se ha impulsado desde la perspectiva de la alfabetización digital (en entornos educativos formales), la cual se enfoca en el desarrollo de habilidades, capacidades y conocimientos necesarios para utilizar las TIC tales como: el manejo del hardware y software; las habilidades de búsqueda, clasificación, evaluación y presentación de la información; y el uso de aplicaciones específicas para el entorno laboral (Castaño, 2016). Pero en estos ejercicios alfabetizadores, la reflexión sobre los procesos de aprendizaje mediados por el género en torno a las tecnologías digitales y las relaciones de poder implícitas en los mismos permanece ausente.

Diferentes autoras han señalado que el aprendizaje en, por y para las tecnologías está mediado por el género, lo que se traduce en una construcción sociocultural de la tecnología digital como un ámbito propio de la masculinidad y en una socialización digital diferenciada (De Sanmamed, Flecha \& Elboj, 2002; Vergés, Hache \& Cruells, 2011; Natansohn, 2013; Rebollo-Catalán, Pérez y Vico-Bosch, 2015; Becerril Martínez, 2018 a). En los entornos educativos formales, de acuerdo con la UNESCO, los estereotipos de género que caracterizan a las mujeres como incompetentes, torpes y poco interesadas en las tecnologías provocan que no se fomente su participación en la producción y manejo 
de las mismas. Además, sus prácticas digitales se clasifican como 'básicas' y 'poco productivas' len oposición a las prácticas especializadas y eficaces atribuidas a los varones) lo cual las excluye de participar en los procesos de innovación, ya que sus hábitos, necesidades y dinámicas no son tomadas en cuenta. Esto explica que, a lo largo de la educación formal, las niñas y jóvenes no cuenten con las mismas oportunidades para desarrollar habilidades y destrezas tecnológicas, perdiendo interés por este ámbito. Lo anterior repercute en su elección de profesión, en sus oportunidades de ingresar a espacios laborales dependientes de las tecnologías, y en las posibilidades de aprovechar los beneficios de las tecnologías digitales en su vida (UNESCO 2019). Para remediar esta socialización diferenciada en los entornos cercanos, de acuerdo con los trabajos mencionados, las mujeres generan otras trayectorias de aprendizaje que se caracterizan por ser autodidactas, solitarias, intuitivas y basadas en el ensayo-error. Es decir, generan modos propios para incorporarse al uso de las TIC.

Al reconocer que el género estructura los procesos de aprendizaje, la intención de fomentar los conocimientos, habilidades y capacidades tecnológicas de las mujeres es insuficiente porque ataca el resultado de dichos procesos (las diferencias de uso) pero no el factor que constituye las diferencias; lo que se requiere es una re-significación de lo tecnológico.

Por esta razón, más que hablar de alfabetización digital, se considera que el concepto central en los procesos de aprendizaje de las mujeres en, con y para las TIC es la apropiación tecnológica. Ésta se define como la manera en que las personas incorporan la tecnología a sus quehaceres cotidianos, tanto en su dimensión práctica como en su dimensión simbólica" (Becerril, 2018 b, p.75). Estas dos dimensiones que conforman las apropiaciones tecnológicas se entretejen, por lo que cuando hablamos de uso tecnológico, estamos también aludiendo a la carga significativa que éste conlleva" (p.76). En este sentido, el concepto de apropiación tecnológica apunta a la construcción de relaciones con las tecnologías distintas a las impuestas por el orden dominante a partir de los significados que se habilitan, reconociendo la agencia de quienes integran las comunidades usuarias ${ }^{2}$. Para Morales, la apropiación tecnológica implica una reflexión sobre las determinaciones leconómicas, sociales e ideológicas) y sobre los discursos implícitos en las TIC para generar prácticas sociales que "se expresan en el uso competente de esos objetos, su deseo y libertad de adaptarlos creativamente a sus propias necesidades, convicciones o intereses, en el marco de la construcción de proyectos de autonomía individual y colectiva" (2011, p.56).

Por eso, Rosalía Winocour (2007, p.554) dice que la apropiación tecnológica pasa por el uso (donde se activan los conocimientos y habilidades), la socialización (donde se activan las representaciones compartidas de la tecnologíal y la significación (donde se articula el sentido del uso de la tecnología desde determinado marco de representación). Siguiendo esta idea, si la relación de las mujeres con las TIC se ha significado desde la exclusión, los procesos de apropiación son clave para generar significaciones alternas desde otros marcos de sentido. Concretamente, hablar de la apropiación tecnológica desde la perspectiva de género implica colocar la experiencia de las personas (en este caso, de las mujeres como sujetos de génerol en el centro de los procesos de aprendizaje, considerando el conjunto de prácticas, conocimientos, motivaciones, representaciones y significados presentes en su relación con determinadas tecnologías (Becerril, 2018 a).

\subsection{La propuesta de las redes de tecnopolítica feminista.}

Pensar en procesos de apropiación tecnológica con perspectiva de género en entornos educativos formales resulta complicado porque estos espacios reproducen los estereotipos, las prácticas y los modelos de aprendizaje que mantienen la jerarquía de lo masculino asociada a las tecnologías digitales. Por eso, una alternativa a dichos espacios son las organizaciones emergentes que fomentan procesos de apropiación tecnológica de las mujeres desde perspectivas que rescaten el potencial subversivo de las mismas. En este trabajo, se considera que estas organizaciones configuran redes de tecnopolítica feminista, las cuales colocan la estructura y la dinámica de red como eje de los procesos sociales. Sierra y Gravante hablan de tecnopolítica como una mediación transformadora y descentralizada originada por las tecnologías digitales, basada en la democracia del código y la creación colectiva (2017, p.7). Lo que distingue a esta mediación es el aspecto de la reticularidad, 
es decir, la estructura y la dinámica en red como resultado de enlaces cruzados en todos los niveles, donde las actividades difieren entre nodos y agentes de la red pero se desarrollan conjuntamente a partir objetivos compartidos.

A partir de las ideas de autoras como Monserrat Boix (2015), Sonia Reverter (2013) y Guiomar Rovira (2018), aquí se define a la tecnopolítica feminista como una propuesta para generar conexiones entre mujeres, tecnologías y otros agentes que transformen las relaciones de poder implícitas en el orden de género. Así, el eje de la propuesta tecnopolítica feminista no es fomentar los usos de la tecnología, sino politizar las prácticas digitales en la vida cotidiana de las mujeres.

En este sentido, se considera que en las redes de tecnopolítica feminista también subyace una propuesta de aprendizaje en red (networked learning), en su concepción más extensa. Goodyear, Banks y McConell (2004) lo definen como una práctica social que a través del uso de las tecnologías de la información promueve las conexiones entre personas, una comunidad y sus recursos de aprendizaje, permitiendo el logro de objetivos cognitivos individuales y colectivos. Para estos autores, el uso de materiales en línea no es una característica suficiente para definir el aprendizaje en red les decir, no se reduce a la educación en líneal sino que el componente central es la interacción:

“La centralidad de la interacción humana, en nuestra concepción del aprendizaje en red, conlleva algunos compromisos pedagógicos y creencias sobre el aprendizaje. En resumen, no tiene sentido el aprendizaje en red si no valora el aprendizaje a través de la cooperación, la colaboración, el diálogo y / o la participación en una comunidad" (2004, p.2).

Ampliando esta noción, Chris Jones (2008) señala que el aprendizaje en red surge de las relaciones de los sujetos sociales con prácticas ubicadas en contextos de acción constituidos por diferentes participantes y en condiciones materiales discernibles: "el aprendizaje en este sentido tiene lugar a lo largo del tiempo, se ubica en espacios tanto de material (incluido el virtual) como social y forma parte de una gama más amplia de prácticas sociales (...) mediadas por una variedad de formas tecnológicas" (Jones, 2008, p.619). Así, el aprendizaje en red como práctica social implica el desarrollo de prácticas en línea y fuera de línea; no es un proceso sólo referenciado a las tecnologías digitales, sino también (y sobre todo) a los procesos habilitados por dichas tecnologías:

El valor para el aprendizaje no radica en la tecnología, ni en el contenido suministrado por un servicio central, sino que reside en las propiedades emergentes que surgen a través de la agregación de muchas partes en las que el todo se vuelve mayor que la suma de sus partes (Jones, 2008, p. 622).

De esta forma, la propuesta del aprendizaje en red como práctica social se acerca a la perspectiva tecnopolítica, donde la mediación tecnológica permite conectar las habilidades, conocimientos y experiencias de múltiples agentes para orientarlos a la construcción de otras formas de participación política. Bajo esta caracterización, se ha identificado el trabajo de diversas organizaciones que promueven la apropiación tecnológica de las mujeres desde espacios no formales, sin formatos curriculares, y sin enfocarse en la generación de habilidades tecnológicas para el manejo de programas o dispositivos.

De manera particular, en México se detecta el surgimiento de una red conformada por colectivos feministas y otras organizaciones que han acompañado las diferentes movilizaciones por los derechos de las mujeres durante los últimos años. El desbordante problema de la violencia de género en el país ${ }^{3}$ lque desde el 2013 ha convocado a las mujeres a las calles con los hashtags \#Feminicidioemergencianacional, \#24A, ${ }^{4}$ \#VivasNosQueremos, \#Miprimeracoso, \#SiMeMatan o \#LaCalleEsNuestral, provocó la visibilización de grupos organizados de mujeres que, a través de diferentes actividades, propiciaban el acercamiento a la tecnología. En primera instancia, estos colectivos pretendían dotar a las mujeres de herramientas para la denuncia de la violencia de género, pero se fueron conectando en una red que ha propiciado la apropiación tecnológica a través de procesos que superan tal uso. En consecuencia, una de las principales preguntas que surgen es: ¿qué modalidades y temáticas configuran la red de aprendizaje para fomentar usos, socializaciones y significados que permitan una apropiación tecnológica? 


\section{Marco metodológico}

El corpus de esta investigación fue recopilado en un inicio en forma de base de datos para representar las redes heterogéneas de actores y actantes que conforman la escena tecnopolítica feminista en México, bajo la perspectiva de la Teoría del Actor-Red, (Pedraza \& Rodríguez, 2019). Para crear la base, se compilaron los carteles de difusión de actividades que a través de la red de Facebook realizaban los colectivos más destacados del país: Ciberseguras, Luchadoras y Laboratorio de Interconectividades (Internet Governance Forum, 2017). Ciberseguras es una iniciativa surgida originalmente en México, actualmente con la participación de colectivos de Brasil, Bolivia y Guatemala, que comparten recomendaciones y recursos de seguridad digital a través de talleres y materiales en la web; Luchadoras se dedica a la producción de información con perspectiva de género la través de su sitio webl así como a la realización de talleres sobre TICs y Género; y el Laboratorio de Interconectividades, es una propuesta hackfeminista que trabaja en conjunto con MedialLabMx y Comando Colibrí la propuesta de la autodefensa digital feminista.

De la revisión de carteles de estas tres colectivas, centrada en la identificación de posibles nodos, surgieron otras organizaciones feministas enfocadas en el mismo rubro: Corazón de Robota, Epic Queen, Hacks de Vida, por mencionar algunas. Asimismo, se encontró una presencia importante de otras organizaciones que sin ser feministas participan con la red tecnopolítica: Data Cívica, Derechos Digitales, La Sandia Digital, Article19, Wikimedia y Social TIC.

En total, se capturaron 82 eventos realizados en el periodo del 24 de abril de 2016 al 25 de noviembre de $2018^{5}$. A partir de este corpus, se registró la información de 8 categorías: colectivos, expositores, temáticas, sedes, ubicaciones, modalidades y respaldos les decir, organizaciones que apoyaban los eventos). Además de encontrarse variados indicios de las redes sumergidas con perspectiva de género que acompañan de manera más discreta las movilizaciones en las redes sociodigitales ${ }^{6}$, también emergió significativamente un importante entramado de redes de aprendizaje subyacente. Para describir este entramado, en este análisis se consideraron solo dos de las categorías registradas: temáticas (es decir, el asunto central del evento) y modalidades (es decir, el formato del evento), con el objetivo de explorar la relación entre la apropiación digital y el aprendizaje en red que se ha tejido desde la tecnopolítica feminista en el escenario mexicano.

Con este fin, se diseñó un abordaje metodológico híbrido consistente en la visualización de las interacciones desde el punto de vista del Análisis de Redes Sociales, aunado a la construcción de variables desde la tradición del análisis de redes semánticas (Kim, 2013; Eddington, 2018). Como sabemos, el Análisis de Redes Sociales persigue un enfoque estructural de las relaciones en un conjunto de nodos o actores y vínculos o interacciones (Wasserman \& Faust, 1994); en el caso que se expone en este artículo en relación con la centralidad de grado que indica que a mayor tamaño del nodo en la red mayor número de relaciones, todo bajo la instrucción del algoritmo de espacialización Force Atlas para ubicar relaciones y distancias proporcionales entre los nodos.

A su vez, el análisis de redes semánticas consiste en la aplicación de técnicas analíticas de asociaciones de pares basadas en significado compartido, comúnmente estudiadas en la relación entre palabras, el análisis de contenido o el hallazgo de percepciones sobrepuestas en un texto (Doerfel, 1998), en este caso a partir de la creación de categorías de sentido desde la confluencia de ámbitos y finalidades tanto en las temáticas como en las modalidades.

\section{Resultados}

Los resultados que se presentan a continuación se dividen en dos. Primero, en la categorización de sentido a partir de las redes de relaciones entre modalidades, que nos ayudó a crear las dimensiones Reflexión, Apropiación e Incidencia como ejes de comprensión de la acción conectiva detrás de la movilización tecnopolítica feminista (ver Imagen 1, elaboración propia, en donde las temáticas se presentan en color violeta, las modalidades en anaranjado y las dimensiones de sentido en color negrol. Estas dimensiones emergen de la pregunta: ¿qué tipo de proceso se intenta generar a través del formato del evento? 
Imagen 1. Redes semánticas de Modalidades (Reflexión, Apropiación e Incidencia)

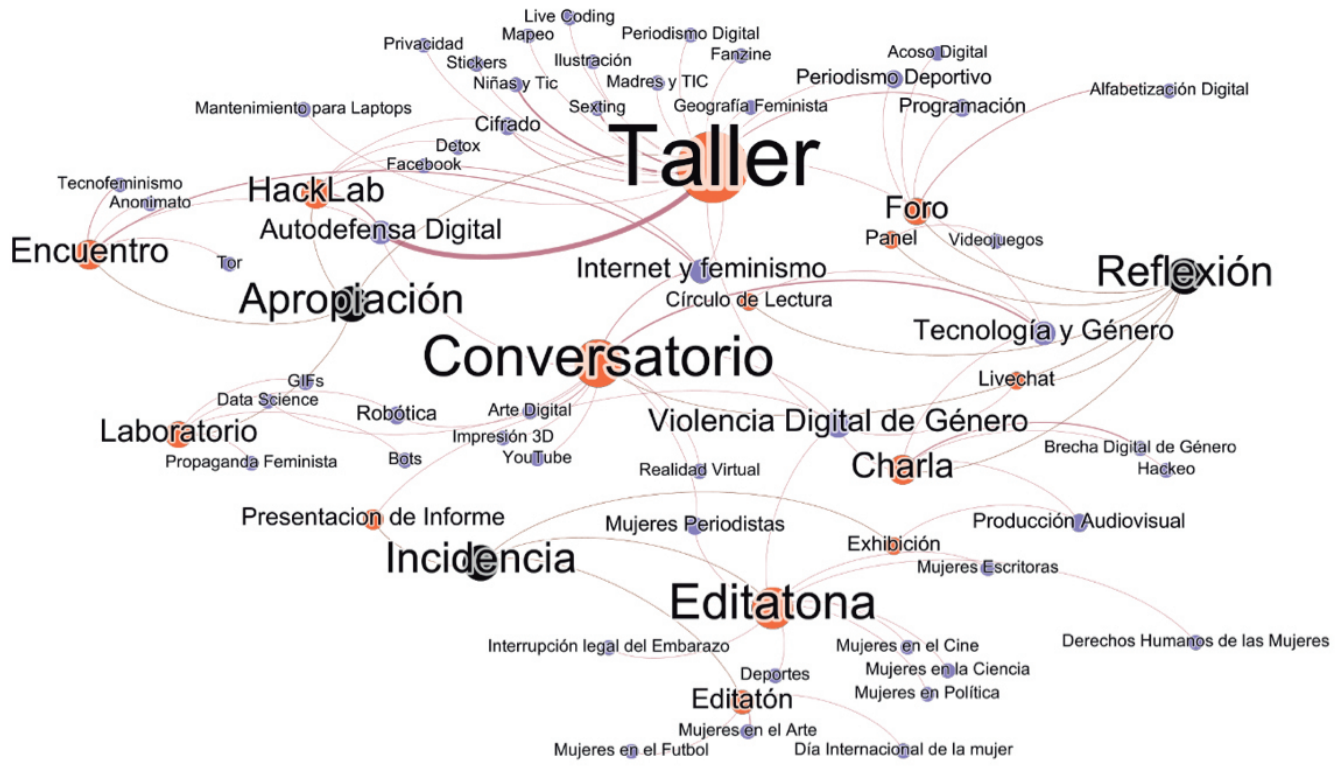

Fuente: Elaboración propia.

Segundo, los hallazgos semánticos en relación con las temáticas, que se aglomeraron alrededor de las siguientes variables, también extraídas por el significado compartido: 1) Reflexión crítica sobre violencia digital, 2) Creación de contenidos, 3) Lenguaje computacional, 4) Perspectiva de género, 5) Prácticas de autodefensa y 6) Formatos de intervención. Estas variables surgen de la pregunta: ¿cuáles son los temas generales en torno a los cuales se organizan estos eventos? Cabe mencionar que en ambos casos se corroboraron las categorías tanto respecto a las modalidades como a las temáticas para fortalecer la pertenencia transversal de sentido.

Respecto a las dimensiones en el plano de las modalidades, los resultados rebasaron el supuesto inicial que era encontrar prácticas de apropiación. En su lugar se obtuvo esta misma dimensión, pero en medio de otras dos que le acompañan muy de cerca: reflexión-que implica el análisis de las relaciones con tecnología sin poner en práctica los aprendizajes-e incidencia -que implica el uso específico de las tecnologías para influir o modificar situaciones concretas. Esto es importante porque supone un esquema de análisis no sobre una sino sobre tres dimensiones de las redes de aprendizaje informal analizadas, como se explica a continuación.
En primer lugar, aparece la dimensión semántica denominada reflexión, basada en las modalidades: Conversatorio, Charla, Foro, Panel, Círculo de lectura y/o Livechat ${ }^{7}$. Enseguida se encuentra la dimensión apropiación acompañada de las modalidades: Taller, Hacklab, Encuentro y Laboratorio. Finalmente, en la dimensión incidencia destacan las modalidades: Editatona, Editatón ${ }^{8}$, Presentación de Informe y Exhibición. En los tres casos el orden de las temáticas se menciona de acuerdo con la mayor centralidad de grado producto del ARS.

Como se intuye, estas tres dimensiones se complementan e incluso hay eventos en los cuales es imposible distinguir cuál aparece con mayor énfasis debido a que más que opciones a elegir se trata de ecosistemas de aprendizaje interconectados que se acompañan con el paso de las espacio-temporalidades. En todo caso, el resultado muestra que el nivel de apropiación es llevado más allá cuando existen prácticas de incidencia o enunciación política más elaborados. Asimismo, no por ello menos importante, la reflexión se convierte en una variable de entrada a las prácticas de apropiación.

En relación con las variables de significado compartido propuestas alrededor de las temáticas, destaca la reflexión crítica sobre violencia digital en el terreno de la dimensión reflexión, en parti- 
Imagen 2. Redes semánticas de la dimensión Apropiación.

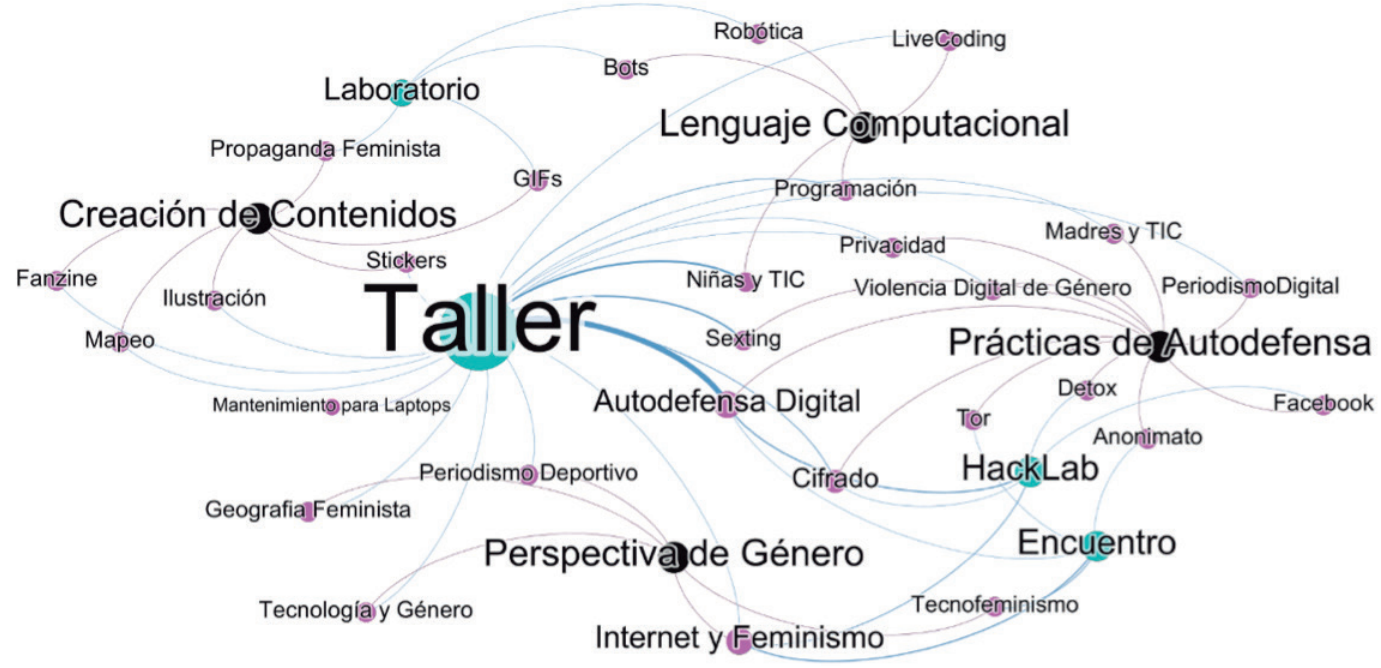

Fuente: Elaboración propia.

cular con las temáticas violencia digital de género y tecnología y género. Ya en la dimensión apropiación aparecen la creación de contenidos (propaganda feminista, gifs, stickers, entre otras), lenguaje computacional (programación, live coding, robótica, bots, entre otras), perspectiva de género (internet y feminismo, tecnofeminismo, geografía feminista, etc.) y prácticas de autodefensa (privacidad, sexting, detox, tor, cifrado, etc.) (ver Imagen 2 , en donde las temáticas son marcadas en color violeta, las modalidades en cian y las variables de sentido en color negrol.
Finalmente, en la dimensión incidencia aparece la red semántica centrada en los formatos de intervención, asociada principalmente a las temáticas violencia digital de género -modalidad presentación de informe- y los diferentes ejercicios de escritura en Wikipedia para disminuir la brecha de aparición de mujeres en disciplinas como el arte, el cine, la política, el fútbol, la ciencia, el periodismo, etc. (ver Tabla 1).

Tabla 1. Dimensiones y Variables semánticas.

\begin{tabular}{|c|c|c|}
\hline Dimensión de sentido & Modalidades & Variables de Sentido (Temáticas) \\
\hline Reflexión & $\begin{array}{l}\text { - Conversatorio } \\
\text { - Charla } \\
\text { - Foro } \\
\text { - Panel } \\
\text { - Círculo de lectura } \\
\text { - Livechat }\end{array}$ & $\begin{array}{l}\text { - Reflexión Crítica sobre } \\
\text { Violencia Digital }\end{array}$ \\
\hline Apropiación & $\begin{array}{l}\text { - Taller } \\
\text { - Encuentro } \\
\text { - Hacklab } \\
\text { - Laboratorio }\end{array}$ & $\begin{array}{l}\text { - Creación de Contenidos } \\
\text { - Lenguaje Computacional } \\
\text { - Perspectiva de Género } \\
\text { - Prácticas de Autodefensa }\end{array}$ \\
\hline Incidencia & $\begin{array}{l}\text { - Editatona } \\
\text { - Editatón } \\
\text { - Presentación de Informe } \\
\text { - Exhibición }\end{array}$ & - Formatos de Intervención \\
\hline
\end{tabular}

Fuente: Elaboración propia. 


\section{Conclusiones}

Aunque el objetivo inicial era analizar la apropiación en la red de colectivos feministas, la aparición de las otras dos dimensiones (reflexión e incidencial a partir de la conexión de modalidades revela relaciones significativas que contribuyen a los procesos de apropiación tecnológica en México. Las modalidades de la dimensión reflexión promueven la socialización y la significación desde una perspectiva dialógica, sin pasar por el uso. El conversatorio lla segunda modalidad más recurrente de la red), charla, panel, y demás modalidades de esta variable tienen como eje la conversación, con el intercambio de experiencias, saberes, dudas, opiniones y propuestas desde la horizontalidad lesto es, aunque suponen la participación de especialistas en los temas, se busca no resaltar la categoría de expertas en el reconocimiento de que todas las aportaciones son valiosas. la conversación, como eje de la socialización de significados que fomentan la reflexión, no busca generar consensos sino ampliar los marcos desde los cuales se producen colectivamente otras significaciones de las tecnologías en la vida de las mujeres.

La relación de las modalidades de la dimensión reflexión con la variable temática reflexión crítica sobre violencia digital indica una preocupación por identificar, compartir experiencias y generar redes en torno a una problemática vital para la población femenina. No obstante, a la par surge la variable temática perspectiva de género, con temas relativos a las diferencias en el uso de los dispositivos, las prácticas y los hábitos digitales; e incluso posturas teóricas y políticas específicas, como el feminismo y el tecnofeminismo, que en sí mismas constituyen marcos de interpretación para la relación de las mujeres y las tecnologías. Por todo esto, aunque en la dimensión Reflexión no existan actividades que promuevan el uso, sí abona a los procesos de apropiación al re-significar prácticas, plataformas, dispositivos y experiencias a través de ejercicios dialógicos.

En la dimensión apropiación se encontraron modalidades orientadas a promover el uso laprendizajes de programas, dispositivos o plataformas), enlazadas a un nivel de subjetivación colectiva (donde los aprendizajes se generan en acompañamiento) y que permiten un proceso de re-significación que transforma la posición de exclusión femenina de las tecnologías digitales. En las modalidades de apropiación se reconoce la agencia de las mujeres, ya que están centradas en el hacer, experimentar 0 fabricar.

Una primera relación significativa surge de la modalidad taller (como espacio colectivo, creativo y, horizontall y la variable temática creación de contenidos. Esta relación indica una propuesta de aprendizaje encaminada a la generación de productos comunicativos: fanzines, gifs, ilustraciones, mapas, videos, etc. En este sentido, subyace otro propósito: convertir a las mujeres en emisoras, con mensajes propios, producidos con las tecnologías digitales.

Otro nodo destacado es prácticas de autodefensa, como una variable de sentido temático que emerge ligada a la variable reflexión crítica sobre violencia digital (el tema central de la dimensión reflexión). Aquí se interpreta que generar conocimientos en torno a los temas de seguridad digital, cifrado, anonimato o privacidad contribuye a prevenir, disminuir o bloquear las prácticas de violencia digital que enfrentan las mujeres. Dichos conocimientos incluyen estrategias para el uso de plataformas de uso cotidiano (como Facebook), programas de navegación segura (como Tor) o herramientas para grupos específicos (madres, periodistas, activistas).

Dos temas merecen mención especial: autodefensa digital como una propuesta feminista que busca transitar del concepto de riesgo al concepto de prevención de las violencias; y sexting seguro, que además de conocimientos sobre seguridad digital supone un proceso de apropiación del cuerpo, la sexualidad y el derecho al goce. Así, en este nodo se promueven usos, socializaciones y significaciones que reivindican el derecho a participar en las TIC: frente a las violencias que buscan excluirlas de los entornos digitales, las mujeres transforman su posición de víctimas para mantenerse como participantes activas.

La variable temática prácticas de autodefensa aparece vinculada a la modalidad de HackLab o laboratorio hacker feminista, lo que implica procesos de apropiación con características específicas: a) el uso de software libre, de códigos y licencias abiertas procedentes del paradigma de la cultura libre de internet, que pugna por la autonomía 
tecnológica fuera de las lógicas de las empresas dominantes; b) la socialización desde la ética hacker, que coloca el gozo en el centro del uso de las tecnologías; y cl la recuperación del significado de hackear como usar las tecnologías en modos transgresores, creativos, con propósitos distintos para los que fueron diseñadas. Encontrar una propuesta que les permita a las mujeres la autonomía, el gozo y la transgresión habla de una apropiación opuesta a los usuales procesos de socialización de las tecnologías.

En este sentido, una última relación significativa es la de los HackLabs con la categoría temática lenguaje computacional, que incluye temas que se consideran de alta especialización como programación, robótica, bots o livecoding. Estos temas conllevan el desafío de manejar el lenguaje de los dispositivos tecnológicos, de ordenar las actividades que éstos realizan, y en cierta medida, de dominar las tecnologías. Que en esta variable temática se encuentren actividades dirigidas a las niñas constituye el punto más radical de la propuesta de apropiación, porque apunta a impedir desde temprana edad la exclusión de las tecnologías a nivel simbólico, práctico y cognitivo.

Así, las temáticas y modalidades de la red de apropiación se ligan con las posibilidades de participar de otra manera en los entornos digitales, en rubros como el arte, el periodismo e incluso el activismo. Un indicador de esto es la aparición en la red de la dimensión incidencia, donde se encuentran actividades con resultados significativos no solo para las mujeres que participan en su proceso de producción sino para personas en general dentro y fuera de los entornos digitales: exhibición de producciones audiovisuales que son difundidas ampliamente, informes sobre problemáticas específicas, o la creación de contenidos para la web. En este sentido, la Editatona, que se realiza con el propósito de aumentar las entradas sobre mujeres en Wikipedia, se consideran la modalidad más completa dentro de la red de aprendizaje: implica una reflexión sobre la desigualdad de género en contenidos en los sitios web; genera procesos de apropiación tecnológica para el uso de una plataforma de creación de contenidos, con el aprendizaje de reglas de edición y de escritura enciclopédica; e incide directamente en Internet, al aumentar la cantidad y calidad de referencias informativas con perspectiva de género en la enciclopedia más consultada del mundo. Destaca también la presentación y publicación del informe "La violencia en línea contra las mujeres en México", presentado a la Relatora sobre la violencia contra las mujeres de la Organización de las Naciones Unidas en noviembre de 2017; este informe, además de la relevancia del tema, constituye el primer esfuerzo documental realizado por esta red de colectivos de tecnopolítica feminista, en colaboración con organizaciones internacionales como la Asociación para el Progreso de las Comunicaciones (APC) y la propia ONU.

En conclusión, el análisis de las redes de aprendizaje que se conforman durante el trabajo de los colectivos de tecnopolítica feminista en México revela una propuesta que busca transformar la relación de las mujeres con las Tecnologías de la Información y la Comunicación a través de su uso, socialización y significación. En específico, con la aparición de un ecosistema de interrelaciones de reflexión, apropiación e incidencia entre temáticas y modalidades que posibilita otras formas de participación política entendidas como nuevos marcos de sentido con perspectiva de género.

\section{Notas}

1 Además del género, la brecha digital supone otras categorías sociales que determinan las diferencias en la participación en las TIC, como la edad, el nivel socioeconómico, la clase, la localización geográfica, etc. Estas categorías son interseccionales, por lo cual, las brechas aumentan dependiendo de la ubicación de las personas en más de una de ellas.

2 Aquí se recupera el concepto de agencia de Sherry Ortner (1984, p.126), quien lo define como la capacidad de los sujetos sociales de transformar activamente sus condiciones estructurales.

3 Según la Encuesta Nacional sobre la Dinámica de las Relaciones en los Hogares (INEGI, 2015), se estima que 6 de cada 10 mujeres mayores de 15 años de edad han sido víctimas de violencia de género. Según la ONU Mujeres (2018), cada día son asesinadas 9 mujeres en el país. Estas estadísticas se han hecho visibles con casos paradigmáticos que reflejan la impunidad en el sistema de justicia, la criminalización de las víctimas de violencia de género por parte de autoridades y medios de comunicación, y la complicidad de las instituciones. Ante este panorama, han aumentado las movilizaciones no institucionales les decir, realizadas en otras fechas que no sean el 8 de marzo por el Día Internacional de la 
Mujer o el 25 de noviembre, por el Día Internacional de la Erradicación de la Violencia contra las Mujeres).

4 Con este hashtag se promovió la Movilización Nacional contra las Violencias Machistas, también denominada “Primavera Violeta", el 24 de abril del 2016, derivada de casos de acoso y violencia sexual que cobraron una notable visibilidad. La marcha se efectuó en más de 40 ciudades, acompañada de testimonios en redes digitales que denunciaban abusos, acosos y violaciones de cientos de mujeres.

5 El 24 de abril de 2016 se efectuó la marcha de la Primavera Violeta, mientras que el 25 de noviembre de 2018 fue el más reciente Día Internacional de la Violencia contra la Mujer, dos fechas que se consideran significativas para enmarcar el periodo de recopilación.
6 En los registros por categorías se distingue 50 temáticas, 37 respaldos, 32 colectivos 125 colectivos que trabajan el tema de tecnologías, 7 que trabajan otros temas), 19 sedes ubicadas en 9 localidades, 14 expositores y 14 modalidades. El análisis del corpus completo se desarrolla en otro artículo (Pedraza \& Rodríguez, 2019)

7 Charlas a través de plataformas digitales, a diferencia de las otras modalidades, que son presenciales

8 Un Editatón es un maratón de edición de artículos de Wikipedia realizado en espacios determinados, donde cualquier persona puede participar. La diferencia con la Editatona radica en que en esta última: al solo participan mujeres; b) solo se editan temas relacionados con las mujeres.

\section{Referencias bibliográficas}

Becerril, W. (2018 a). Mujeres que codean: la apropiación tecnológica de estudiantes egresadas de Laboratoria en México. Controversias y concurrencias latinoamericanas Vol. $10 \mathrm{~N}^{\circ} 16,67-75$.

Becerril, W. (2018 b). Indagar las apropiaciones tecnológicas desde una perspectiva de género feminista. En S. Lago (coord.), Acerca de la apropiación de tecnologías: teoría, estudios y debates (págs. 65-84). Buenos Aires: Del Gato Gris.

Boix, M. (2015). Desde el Ciberfeminismo hacia la Tecnopolítica feminista. Revista Pillku, 18. Disponible en https://pillku.org/article/desde-el-ciberfeminismo-hacia-latecnopolitica-fem/

Braidotti, R. (2002). Un ciberfeminismo diferente. Debats, 76, 100-117.

Castaño, C. (2005). Las mujeres y las tecnologías de la información. Internet y la trama de nuestra vida. Madrid: Instituto Andaluz de la Mujer.

Castaño, C. (2016). La brecha de género en la $4^{\mathrm{a}}$ revolución industrial. Gaceta sindical: reflexión y debate, (27), 97-106.

Castaño, C. ; Caprile, M. (2010). Marco conceptual sobre género y tecnología. En C. Castaño, \& M. Castells (Ed.). Género y TIC. Presencia, posición y políticas (pp. 21-81). Barcelona, España: UOC Ediciones.

De Sanmamed, A., Flecha, F. \& Elboj, C. (2002). Mujeres, aprendizaje dialógico y transformación social. Contextos educativos: Revista de educación, 5, 159-172.

Doerfel, M. L. (1998). What constitutes semantic network analysis? A comparison of research and methodologies. Connections, 21(2), 16-26.

Eddington, S. M. (2018). The Communicative Constitution of Hate Organizations Online: A Semantic Network Analysis of "Make America Great Again”. Social Media+ Society, 4(3), 2056305118790763. 
Goodyear, P., Banks, S. H. \& McConell, D. (2004). Advances in Research on Networked Learning. Londres: Kluwer Academic Publishers.

Gross, B. (2011). Evolución y retos de la Educación virtual. Barcelona: UOC.

Haraway, D. (1995). Ciencia, Cyborgs y mujeres. La reinvención de la naturaleza. Madrid: Cátedra.

Hargittai, E. (2007). Whose Space? Differences Among Users and Non- Users of Social Network Sites. Journal of Computer-Mediated. Digital Media and Masculinity Communication, 13(1), 276-297.

Hawthorne, S., \& Klein, R. (1999). Cyberfeminism. Melbourne: Spinifex Press.

INEGI- Instituto Nacional de Estadistica y Geografía (2015). Encuesta Nacional sobre sobre la Dinámica de las Relaciones en los Hogares. Ciudad de México: Instituto Nacional de Estadística y Geografía.

Internet Governance Forum (2017). Latin America in a Glimpse: Gender, Feminism an Internet in Latin America. Suiza: Derechos Digitales/APC.

Jones, C. (2008). Networked Learning-a social practice perspective. En Proceedings of the 6th International Conference on Networked Learning (pp. 616-623). Lancaster: Lancaster university.

Kim, L. (2013). Denotation and connotation in public representation: semantic network analysis of Hwang supporters' internet dialogues. Public understanding of science, 22(3), 335-350.

Maffía, D. (2017). Epistemología feminista: la subversión semiótica de las mujeres en la ciencia. Revista Venezolana de Estudios de la Mujer, 12-(28)., 63-98.

Morales, S. (2011) “Acceso y apropiación de Tecnologías de la información y la comunicación. Una apuesta de política pública en educación.”. En Cabello, R. \& Morales, S. (eds.) Enseñar con tecnologías. Nuevas miradas en la formación docente (pp.47-75). Buenos Aires: Prometeo.

Natansohn, G. (2014). Por una agenda feminista para Internet y las comunicaciones digitales. III Congreso Género y Sociedad, Voces, cuerpos y derechos en disputa. Córdoba: Universidad Nacional de Córdoba.

Natasohn, G. (2013). Internet en código femenino. Teorías y prácticas. Buenos Aires: La Crujía.

Núñez, S. (2011). Activismo y colectivos en red: praxis feminista y violencia de género. Asparkía. Investigación feminista, 85-98.

ONU. (2015). Informe final del Grupo de Trabajo sobre Género de la Comisión de Banda Ancha. Nueva York: Comisión de las Naciones Unidas para la Banda Ancha-UITUNESCO.

ONU-Mujeres. (2018). Comunicado del Día Internacional de la Erradicación de la Violencia contra la Mujer. Ciudad de México: ONU Mujeres. 
Ortiz Henderson, G. y Garay Cruz, L. M.. (2015). Comunicación, cultura y educación: nueve aproximaciones al estudio de las tecnologías digitales. Ciudad de México: Universidad Autónoma Metropolitana y Juan Pablos Editor.

Ortner, S. (1984). Theory in Anthropology since the Sixties. Comparative Studies in Society and History 26(1), 126-165.

Pagola, L. (2010). Sensibilizaciòn tecnológica, mujeres construyendo la sociedad del conocimiento. En S. Chaher, \& S. Santori, Las palabras tienen sexo II. Herramientas para un periodismo de género (pp. 73-91). Buenos Aires: Artemisa Comunicaciones.

Pedraza C. \& Rodríguez C (2019). Resistencias sumergidas. Cartografía de la Tecnopolítica Feminista en México. (Manuscrito sometido para publicación)

Plant, S. (1998). Ceros + Unos. Mujeres digitales y la Nueva Tecnocultura. Barcelona: Destino.

Rebollo-Catalán, Á., Pérez, R., \& Vico-Bosch, A. (2015). El aprendizaje de las mujeres de las redes sociales y su incidencia en la competencia digital. Prisma Social: revista de investigación social, 15, 122-146.

Reverter, S. (2013). Ciberfeminismo: de virtual a político. Teknocultura, 451-461.

Reverter, S. (2001). Reflexiones en torno al Ciberfeminismo. Asparkía: investigació feminista, (12), 35-51.

Rovira, G. (2018). El devenir feminista de la acción colectiva: las redes digitales y la política de prefiguración de las multitudes conectadas. Teknokultura,15 (2), 223-240.

Sierra, F. \& Gravante, T. (2017). Networks, movements and technopolitics in Latin America. Critical analysis and current challenges. Londres: Palgrave-IAMCR.

UIT. (2017). Mesuring the Information Society Report. Ginebra: Unión Internacional de Telecomunicaciones.

UNESCO. (2019). Descifrar el código: la educación de las niñas y las mujeres en ciencias, tecnología, ingeniería y matemáticas (STEM). Paris: UNESCO.

Vergés, N., Hache, A., \& Cruells, E. (2011). Indagando en la relevancia de Internet en el acceso, uso y deseos de las TIC por parte de las mujeres. Teoría de la Educación. Educación y Cultura en la Sociedad de la Información, 12,(2), 105-121.

Wajcman, J. (2006). El Tecnofeminismo. Valencia: Cátedra.

Wasserman, S. \& Faust, K. (1994). Social network analysis: methods and applications. Cambridge: Cambridge University Press.

Winocour, R. (2007). Nuevas tecnologías y usuarios, la apropiación de las tics en la vida cotidiana cotidiana. Telos: cuadernos de comunicación e inovación, 109-117.

Zafra, R. (2005). Netianas. N(hlacer mujer en Internet. Madrid: Lengua de Trapo. 
- Sobre los autores:

Claudia I. Pedraza Bucio es profesora Investigadora en la Universidad La Salle Ciudad de México. Doctora en Ciencias Políticas y Sociales por la UNAM, con estancias de investigación en la Universidad Autónoma de Barcelona y la Universidad Autónoma Metropolitana. Integrante del Sistema Nacional de Investigadores.

César A. Rodríguez Cano es profesor Investigador en la Universidad Autónoma Metropolitana, unidad Cuajimalpa. Doctor en Ciencias Políticas y Sociales por la UNAM, con estancias de investigación en la Universidad de California en Los Ángeles y la Universidad Iberoamericana. Integrante del Sistema Nacional de Investigadores.

- ¿Como citar?

Pedraza, C. \& Rodríguez, C. (2019). Conversatorios, talleres y editatonas: tecnopolítica feminista y redes de aprendizaje en México. Comunicación y Medios, (40), 84-96. 